\title{
Time-varying continuous and jump betas: The role of firm characteristics and periods of stress
}

\author{
Vitali Alexeev ${ }^{\mathrm{a}, *}$, Mardi Dungey ${ }^{\mathrm{b}}$, Wenying Yao ${ }^{\mathrm{c}}$ \\ ${ }^{a}$ Finance Discipline Group, UTS Business School, University of Technology Sydney, Sydney NSW 2007, Australia \\ ${ }^{b}$ Tasmanian School of Business and Economics, University of Tasmania, Hobart TAS 7001, Australia \\ ${ }^{c}$ Department of Economics, Deakin Business School, Deakin University, Burwood VIC 3125, Australia
}

\begin{abstract}
Using high frequency data we decompose the time-varying beta for stocks into beta for continuous systematic risk and beta for discontinuous systematic risk. Estimated discontinuous betas for S\&P500 constituents over 2003-2011 generally exceed the corresponding continuous betas. Smaller stocks are more sensitive to discontinuities than their larger counterparts, and during periods of financial distress, high leverage stocks are more exposed to systematic risk. Higher credit ratings and lower volatility are each associated with smaller betas. Industry effects are also apparent. We use the estimates to show that discontinuous risk carries a significantly positive premium, but continuous risk does not.
\end{abstract}

Keywords: systematic risk, jumps, equity risk premium, high-frequency data

JEL: C58, G11, G01

*Corresponding author.

Email addresses: Vitali.Alexeev@uts.edu.au, Tel:+61 362262335 (Vitali Alexeev), Mardi.Dungey@utas.edu.au, Tel:+61 362261839 (Mardi Dungey), Wenying.Yao@utas.edu.au, Tel:+61 362267141 (Wenying Yao) 


\section{Introduction}

In the corporate finance literature the cost of equity capital is often determined by CAPM betas. Given that there is now substantial evidence that asset prices evolve as a combination of Brownian motion with stochastic volatility and a jump process, we examine differences between betas calculated for the continuous and jump components of systematic risk. ${ }^{1}$ Unlike the betas routinely calculated for Fama-French factors, our continuous and jump risk betas are directly scale comparable and clearly indicate that for S\&P500 constituent stocks jump betas are substantially higher than continuous betas.

This paper takes advantage of the recent methodology of Todorov and Bollerslev (2010) to construct estimates of beta using high frequency intra-day data. We produce estimates of the extent to which individual firms follow the continuous component of the market - continuous beta; and the extent to which they follow disruptions - discontinuous beta. Using Monte Carlo simulation, we provide the first finite sample performances of the Todorov and Bollerslev (2010) estimation techniques to support our analysis. For a sample of over 500 stocks drawn from the historical constituents of the S\&P500 index, continuous beta is estimated as lower than the jump beta nearly 90 percent of the time. Overall jump betas are 38 percent higher than continuous betas on average. This is more than twice the increase in betas estimated around individual earnings releases by Patton and Verardo (2012). Patton and Verardo (2012) hypothesise that markets learn from earnings announcements about the state of the economy; as jumps are commonly associated with news arrival, a jump beta which exceeds continuous beta may imply that stocks update faster to unexpected information arrival than has been previously estimated. Neither jump nor continuous beta estimates show any obvious trend over the sample period from 2003 to 2011. However, many stocks experienced a significant change in their relationship to the continuous component of systematic market risk during September-October 2008, the time of the Lehman Brothers collapse, rescue of AIG and subsequent implementation of TARP (Troubled Asset Relief Program).

For investors, the knowledge that individual stocks respond differently to the continuous and jump components of systematic risk is likely to provide a valuable tool in managing portfolio diversification. We find that small-sized firms are more sensitive to jumps than their larger counterparts. During periods of financial distress, highly leveraged stocks are more exposed to market jumps, whereas stocks with low leverage react less to market jumps. Stocks with high credit ratings have lower beta, both continuous and discontinuous. The presence of idiosyncratic volatility is associated with higher beta, and more volatile discontinuous beta.

Across different industry categories the response to the continuous component of systematic risk varies considerably, from the lowest sustained level in our sample in the Healthcare and Consumer

\footnotetext{
${ }^{1}$ Confirmation of continuous and jump processes for stocks may be found in Aït-Sahalia and Jacod (2012); the existence of jumps in many assets and the relationship of jumps with news is well-documented in Andersen et al. (2007), Dungey et al. (2009), Jacod and Todorov (2009) and Lahaye et al. (2011). Accounting for jumps has been shown to improve both the estimation of the yield curve (Lahaye et al., 2005), estimates and forecasts of daily volatility (Andersen et al., 2003; Blair et al., 2001) and optimal hedging strategies Lai and Sheu (2010).
} 
Goods sectors, and the highest in the Basic Materials, Technology and Financial sectors. Given our sample period, this latter result is not surprising. Drawing a parallel with the CAPM framework, assets that are least influenced by market fluctuations are said to exhibit defensive characteristics (that is they have defensive betas), while stocks that are most affected can be said to have aggressive betas. The Financial sector exhibits defensive characteristics before the first indications of stress in July 2007, but sharply changes to the most aggressive sector during the crisis. The Basic Materials sector is more exposed to the continuous risk component than the jump risk, a feature unique to this sector. Overall, Technology (in the pre-crisis period), Financial (in the crisis period), Basic Materials and Services are the leading sectors amongst the most aggressive stocks. Healthcare, Consumer Goods and Financial (in the pre-crisis period) prevail among the most defensive stocks.

We further explore the relationship between the two systematic risk components and the expected stock returns using the Fama and MacBeth (1973) cross-sectional regressions. Our results are mixed, and are not completely consistent with theoretical predictions. However, we find broad evidence that the risk premia on continuous risk are insignificant for the majority of sectors, but that there are significantly positive risk premia for discontinuous, or jump, risk. Further decomposition reveals that significant risk premia exist for both the continuous and discontinuous risk prior to the financial crisis, but on average these become insignificant in the crisis period. Similar findings have been obtained in a contemporaneous study by Bollerslev et al. (2016). It should be noted, however, the sampling strategy is different from that of Bollerslev et al. (2016). Focusing on cross-sectional estimation, Bollerslev et al. (2016) consider a sequence of rolling windows to obtain a sample for each window and do not require those samples to be consistent across time. ${ }^{2}$

The remainder of this paper is organised as follows. Section 2 introduces the modelling framework. Section 3 presents new analysis on the performance of jump and continuous beta estimators in finite samples. Section 4 describe the data set and parameter choices used in the estimation. The empirical analysis of the two beta estimates are presented in Section 5. Section 6 uses Fama and MacBeth (1973) two-stage regressions to estimate the risk premia on these two risk components. Section 7 concludes. Robustness analyses are performed in Appendix A and Appendix C.

\section{Modeling Framework}

A standard market model regression relating returns on an individual stock, $r_{i}$, to a benchmark market return, $r_{0}$, takes the form,

$$
r_{i}=\alpha_{i}+\beta_{i} r_{0}+\epsilon_{i}, \quad i=1, \ldots, N
$$

\footnotetext{
${ }^{2}$ The present paper deals with the changing nature of estimates of continuous and discontinuous betas for the same stocks and thus considers only liquid stocks with data availability for the most of the sample period. In both cases the sample selection is appropriate for the research question at hand: Bollerslev et al. (2016) favour a 75 minute sampling frequency to overcome the lack of liquidity across a larger cross section of stocks, whilst we use a 5 minute sampling frequency which gives us sample size advantages in our time series analysis.
} 
The $\beta_{i}$ coefficient in equation (1) is the sensitivity of the expected return on the $i$-th asset to the return on the market (or systematic) factor. The classic reference in the literature is Sharpe (1963). Todorov and Bollerslev (2010) show how, using high frequency data, the return can be decomposed into two components: one associated with continuous price movements and the other associated with jumps. Hence in the presence of both components, equation (1) becomes

$$
r_{i}=\alpha_{i}+\beta_{i}^{c} r_{0}^{c}+\beta_{i}^{d} r_{0}^{d}+\epsilon_{i}, \quad i=1, \ldots, N,
$$

where $r_{0}=r_{0}^{c}+r_{0}^{d}$. Using equation (2) we can attribute the overall systematic risk to either the continuous component $r_{0}^{c}$, or the discontinuous component $r_{0}^{d}$. Recognition of this is important as the implication that $\beta_{0}^{c}=\beta_{0}^{d}=1$ is critical in the identification of the $\beta_{i}^{c}$ and $\beta_{i}^{d}$ coefficients in recent Todorov and Bollerslev (2010).

The log-price of any asset $p_{t}$ is usually assumed to follow a continuous-time jump diffusion process, and hence, omitting an asset $i$ subscript without loss of generality,

$$
r_{t} \equiv \mathrm{d} p_{t}=\alpha_{t} \mathrm{~d} t+\sigma_{t} \mathrm{~d} W_{t}+\kappa_{t} \mathrm{~d} \mu_{t}, \quad t \in(0, T),
$$

where $W_{t}$ is a standard Brownian motion, $\sigma_{t}$ denotes the stochastic volatility, $\mu_{t}$ is a counting process for the discrete jump component, and $\kappa_{t}=p_{t}-p_{t-}$ denotes the size of the jump at time $t$. The usual quadratic variation for this process is defined as

$$
[r, r]_{t}^{2}=\int_{0}^{t} \sigma_{s}^{2} \mathrm{~d} s+\sum_{0<s \leq t} \kappa_{s}^{2}
$$

Linking equations (2) and (3) together for $i=0$, the market portfolio returns can be defined as

$$
r_{0, t}=\mathrm{d} p_{0, t}=\alpha_{0, t} \mathrm{~d} t+\sigma_{0, t} \mathrm{~d} W_{0, t}+\kappa_{0, t} \mathrm{~d} \mu_{0, t}
$$

and for any asset $i=1, \ldots, N$,

$$
r_{i, t}=\alpha_{i, t} \mathrm{~d} t+\beta_{i}^{c} \sigma_{0, t} \mathrm{~d} W_{0, t}+\beta_{i}^{d} \kappa_{0, t} \mathrm{~d} \mu_{0, t}+\epsilon_{i}
$$

where $\epsilon_{i}$ contains the idiosyncratic movements in each individual asset price $p_{i, t}$, both continuous and jumps. It follows that the quadratic covariation between $r_{i}$ and $r_{0}$ is

$$
\left[r_{i}, r_{0}\right]_{t}^{2}=\beta_{i}^{c} \int_{0}^{t} \sigma_{0, s}^{2} \mathrm{~d} s+\beta_{i}^{d} \sum_{0<s \leq t} \kappa_{0, s}^{2} .
$$

If we are able to separate the continuous component of (7), $\left[r_{i}^{c}, r_{0}^{c}\right]_{t}=\beta_{i}^{c} \int_{0}^{t} \sigma_{0, s}^{2} \mathrm{~d} s$, then $\beta_{i}^{c}$ can be represented as

$$
\beta_{i}^{c}=\frac{\left[r_{i}^{c}, r_{0}^{c}\right]_{t}^{2}}{\left[r_{0}^{c}, r_{0}^{c}\right]_{t}^{2}} \quad, i=1, \ldots, N
$$

For the jump beta, Todorov and Bollerslev (2010) suggest using higher power covariation of the 
discontinuous component, $\left[r_{i}^{d}, r_{0}^{d}\right]_{t}^{2 \tau}$. It is defined as

$$
\left[r_{i}^{d}, r_{0}^{d}\right]_{t}^{2 \tau}=\left(\beta_{i}^{d}\right)^{\tau} \sum_{0<s \leq t} \kappa_{0, s}^{2 \tau}=\left(\beta_{i}^{d}\right)^{\tau}\left[r_{0}^{d}, r_{0}^{d}\right]_{t}^{2 \tau}, \quad \tau \geq 1
$$

which will lead to a representation for $\beta_{i}^{d}$.

\subsection{The Estimators in Discrete Time}

In practice, we usually observe prices and returns every $\Delta$ time interval, from $0, \Delta, 2 \Delta, \ldots$, to $[T / \Delta] \cdot \Delta$. Keeping $\Delta$ fixed, we denote the $\Delta$-period return on asset $i$ by $r_{i, j}=p_{i, j \Delta}-p_{i,(j-1) \Delta}$, for $i=0,1, \ldots, N$, and $j=1, \ldots,[T / \Delta]$. Using vector notation, let the $(N+1) \times 1$ vector of the observed returns be $\mathbf{r}_{j}=\left(r_{0, j}, r_{1, j}, \ldots, r_{N, j}\right)^{\prime}$.

The consistent estimators for $\beta_{i}^{c}$ and $\beta_{i}^{d}$ given by Todorov and Bollerslev (2010) are constructed as follows. We set a truncation threshold $\boldsymbol{\theta}=\left(a_{0} \Delta^{\mathscr{\omega}}, a_{1} \Delta^{\omega}, \ldots, a_{N} \Delta^{\mathscr{\omega}}\right)^{\prime}$, where $\mathcal{\omega} \in\left(0, \frac{1}{2}\right)$, and $a_{i} \geq 0$, $i=0, \ldots, N$. We allow for different truncation thresholds across different assets by controlling $a_{i}{ }^{3}$ The continuous price movement corresponds to those observations that satisfy $\left|\mathbf{r}_{j}\right| \leq \boldsymbol{\theta}$. The discrete-time estimator of the continuous beta, $\hat{\beta}_{i}^{c}$, is constructed using the sample counterpart of (8):

$$
\hat{\beta}_{i}^{c}=\frac{\sum_{j=1}^{[T / \Delta]} r_{i, j} r_{0, j} \mathbb{1}_{\left\{\left|\mathbf{r}_{j}\right| \leq \boldsymbol{\theta}\right\}}}{\sum_{j=1}^{[T / \Delta]} r_{0, j}^{2} \mathbb{1}_{\left\{\left|\mathbf{r}_{j}\right| \leq \boldsymbol{\theta}\right\}}}, \quad \text { for } \quad i=1, \ldots, N,
$$

where $\mathbb{1}$ is the indicator function,

$$
\mathbb{1}_{\left\{\left|\mathbf{r}_{j}\right| \leq \boldsymbol{\theta}\right\}}=\left\{\begin{array}{ll}
1 & \text { if }\left|\mathbf{r}_{j}\right| \leq \boldsymbol{\theta} \\
0 & \text { otherwise }
\end{array} .\right.
$$

The discrete time estimator of $\beta_{i}^{d}$ is

$$
\hat{\beta}_{i}^{d}=\operatorname{sign}\left\{\sum_{j=1}^{[T / \Delta]} \operatorname{sign}\left\{r_{i, j} r_{0, j}\right\}\left|r_{i, j} r_{0, j}\right|^{\tau}\right\} \times\left(\frac{\sum_{j=1}^{[T / \Delta]} \operatorname{sign}\left\{r_{i, j} r_{0, j}\right\}\left|r_{i, j} r_{0, j}\right|^{\tau}}{\sum_{j=1}^{[T / \Delta]} r_{0, j}^{2 \tau}}\right)^{\frac{1}{\tau}}
$$

where the power $\tau$ is restricted to $\tau \geq 2$ so that the continuous price movements do not matter asymptotically (see Todorov and Bollerslev, 2010, for more details). The sign in (12) is taken to recover the signs of the jump betas that are eliminated when taking absolute values. The estimator in (12) converges to $\beta_{i}^{d}$ when there is at least one systematic jump (in the market portfolio) on $(0, T]$. Therefore, in order to calculate $\hat{\beta}_{i}^{d}$, we first need to test for the existence of jumps on the log-price series $p_{0}$ of the market portfolio.

\footnotetext{
${ }^{3}$ The literature implements $a_{i}$ as a multiple of the standard deviations of the Brownian component (Ait-Sahalia and Jacod, 2012; Todorov and Bollerslev, 2010), which provides an intuitive interpretation that, for instance, when $a_{i}=3$, price increments that are larger than three standard deviations are classified as jumps.
} 


\subsection{Testing for Jumps}

We use the test statistic proposed by Barndorff-Nielsen and Shephard (2006) to detect jumps in the market portfolio, which is part of the design of the underlying decomposition in the methodology of Todorov and Bollerslev (2010). ${ }^{4}$ The realized volatility at sampling frequency $\Delta$ is defined as

$$
R V_{0}=\sum_{j=1}^{[T / \Delta]} r_{0, j}^{2} \stackrel{p}{\longrightarrow}\left[r_{0}, r_{0}\right]_{T}^{2} \quad \text { as } \quad \Delta \rightarrow 0,
$$

where $\left[r_{0}, r_{0}\right]_{T}^{2}$ is the quadratic variation as defined in equation (4). Let the realized bipower variation be

$$
B V_{0}=\sum_{j=1}^{[T / \Delta]-1}\left|r_{0, j}\right|\left|r_{0, j+1}\right| \stackrel{p}{\longrightarrow} \mu_{1}^{2} \int_{0}^{T} \sigma_{s}^{2} \mathrm{~d} s \quad \text { as } \quad \Delta \rightarrow 0,
$$

where $\mu_{1}=\sqrt{2 / \pi}$. Combining equations (4), (13) and (14) implies that

$$
R V_{0}-\mu_{1}^{-2} B V_{0} \stackrel{p}{\longrightarrow} \sum_{0<s \leq T} \kappa_{s}^{2} .
$$

Barndorff-Nielsen and Shephard (2006) suggest using a standardized version of (15) to detect jumps. The feasible test statistic is given by

$$
\hat{\mathcal{J}}=\frac{1}{\sqrt{\Delta}} \cdot \frac{1}{\sqrt{\phi \cdot \max \left(1 / T, D V_{0} / B V_{0}^{2}\right)}}\left(\frac{\mu_{1}^{-2} \cdot B V_{0}-R V_{0}}{R V_{0}}\right) \stackrel{L}{\longrightarrow} \mathcal{N}(0,1),
$$

where $\phi=\pi^{2} / 4+\pi-5$, and $D V_{0}$ is the realized quadpower variation

$$
D V_{0}=\sum_{j=1}^{[T / \Delta]-3}\left|r_{0, j}\right|\left|r_{0, j+1}\right|\left|r_{0, j+2}\right|\left|r_{0, j+3}\right| \text {. }
$$

\section{Finite Sample Simulation}

Todorov and Bollerslev (2010) derive the asymptotic properties of the estimators presented for $\beta^{c}$ and $\beta^{d}$. However, before implementing the practical example of this paper we consider the issue of finite sample behaviour when faced with alternative sampling frequencies and potentially large discrepancies between the values of $\beta^{c}$ and $\beta^{d}$.

We simulate the market return according to equation (5) with jump intensity, $\mu_{0}$, set to 3 and the size of the jumps, $\kappa_{0, t}$, drawn from a normal distribution $\mathcal{N}\left(0.1,0.15^{2}\right)$. Since the estimation of the discontinuous beta requires the existence of at least one market jump, we ensure that each simulated sample path of the market portfolio contains at least one jump. We then simulate the returns for each asset using equation (6) across combinations of the continuous and the discontinuous beta,

\footnotetext{
${ }^{4}$ Although the jump test by Barndorff-Nielsen and Shephard (2006) is known to suffer from size and power distortion in small samples, we follow existing literature and use rather restrictive critical values to avoid falsely identifying jumps.
} 
ranging from 0.1 to 2.0 with 0.1 increments. We simulate 1,000 time series of asset returns for each combination of $\beta^{c}$ and $\beta^{d}$. ${ }^{5}$

Since our empirical application will consider monthly beta estimates for equity markets, we consider samples consistent with 10-minute, 5-minute and 30-second data sampling for equidistant price observations between 9:30am and 4:00pm each trading day excluding overnight returns, resulting in simulated lengths of 809, 1617 and 16170 observations respectively. We focus on the simulation with 5-min data and contrast these results with the estimated finite sample bias for the other sampling frequencies.

Figure 1 depicts the average finite sample estimation bias for continuous and discontinuous beta estimates as we vary the values of $\beta^{c}$ and $\beta^{d}$ in the true data generating process (DGP). Specifically, panels on the left represent the difference between the average estimated continuous beta and the true continuous beta, $\hat{\beta}^{c}-\beta^{c}$, over 1,000 simulations. The true values of $\beta^{c}$ are given on the horizontal axis and true values of $\beta^{d}$ on the vertical axis. Similarly, the panels on the right show the difference between the average estimated discontinuous beta and the true discontinuous beta, $\hat{\beta}^{d}-\beta^{d}$. The colour-scale to the right of each panel indicates the magnitude of the estimation error. The results show that the estimator of $\beta^{c}$ from equation (10) performs well across any combination of $\beta^{c}$ and $\beta^{d}$. The difference between $\hat{\beta}^{c}$ and $\beta^{c}$ is always below 0.01 for the 5-min simulated samples; this bias is negligible compared with the magnitude of the true continuous betas. The magnitude of the estimation error decreases as sample size increases (down the left hand panel of the figure) supporting the asymptotic consistency of the estimated $\beta^{c}$.

On the other hand, bias in the estimate of the discontinuous beta, $\beta^{d}$, is more substantial. For example, for 10-minute data, the bottom right corner of Panel (b) shows that the bias can be as large as 1.95 when the parameter values are $\beta^{c}=2$ and $\beta^{d}=0.1$ in the true DGP, and -1.72 when $\beta^{c}=0.1$ and $\beta^{d}=2$ (the top left corner). In Panels (d) and (f), results for the same corner observations indicate biases of 1.35 and -0.54 for 5 -min data and 0.44 and -0.05 for 30 -sec data. Thus, as the sample size increases, the magnitude of the estimation error reduces.

Figure 1 shows that the estimation error in $\hat{\beta}^{d}$ is affected by the discrepancy between the true values of $\beta^{c}$ and $\beta^{d}$. When $\beta^{c}$ and $\beta^{d}$ take similar values (i.e. along the 45 degree line in Figure 1 ), the estimation bias in $\hat{\beta}^{d}$ is always close to zero. However, as the difference between $\beta^{c}$ and $\beta^{d}$ becomes larger, the estimation bias increases. Thus, the estimated value of $\beta^{d}$ can be substantially affected by the true value of $\beta^{c}$, as shown in the right hand panels of Figure 1. The importance of this result is that if we expect that the jump beta does differ from continuous beta, the extent of this difference will bias the resulting estimate of the jump beta, making it more difficult to assess arguments as to the relative speed of information absorbtion as posited in Patton and Verardo (2012). To illustrate this point clearly, we plot the estimation bias from the 5-minute simulated samples against the difference between the true values of continuous and jump betas, $\left(\beta^{c}-\beta^{d}\right)$ in Figure 2. In Panel

\footnotetext{
${ }^{5}$ We acknowledge that a number of alternative data generating processes could be entertained to incorporate a jump component into the price series (Jarrow and Rosenfeld, 1984; Hanson, 2007). However, Todorov and Bollerslev (2010) note that even if the single-factor structure in (6) does not hold exactly, the $\hat{\beta}_{i}^{c}$ and $\hat{\beta}_{i}^{d}$ in (10) and (12), respectively, still provide meaningful sensitivity of asset $i$ to the diffusive and jump movements in the market portfolio.
} 
Figure 1: Estimation error in $\hat{\beta}^{c}$ and $\hat{\beta}^{d}$ for different combinations of $\beta^{c}$ and $\beta^{d}$ using sample sizes equivalent to the number of $10-\mathrm{min}, 5-\mathrm{min}$ and $30-\mathrm{sec}$ observations in one month period.

(a) Estimation error in $\hat{\beta}^{c}$ with 10-min data

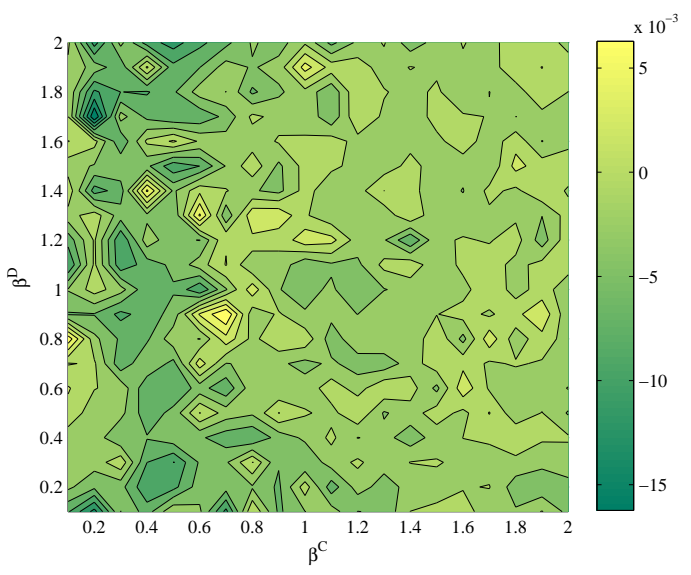

(c) Estimation error in $\hat{\beta}^{c}$ with 5-min data

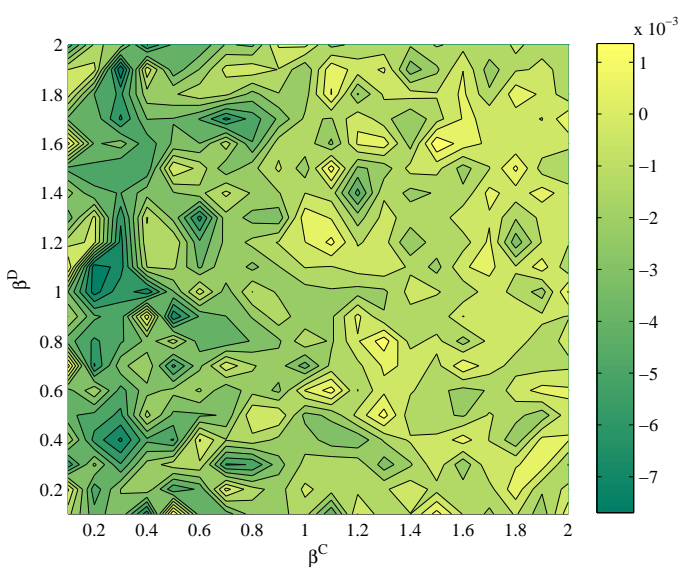

(e) Estimation error in $\hat{\beta}^{c}$ with $30-\sec$ data

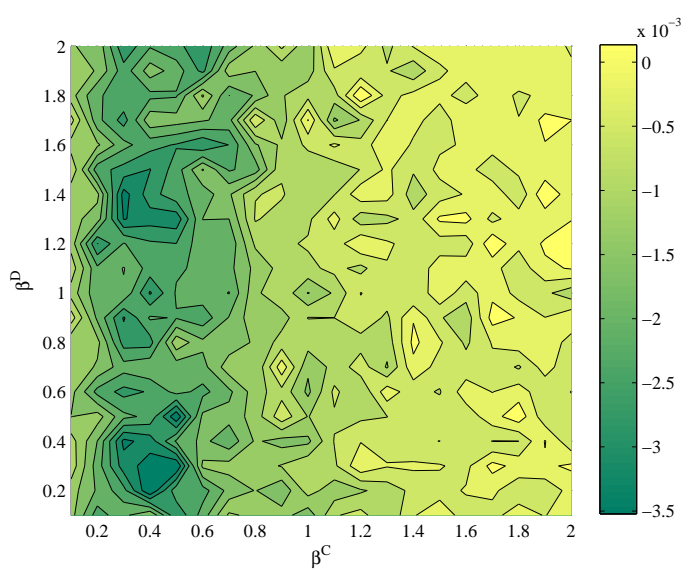

(b) Estimation error in $\hat{\beta}^{d}$ with 10-min data

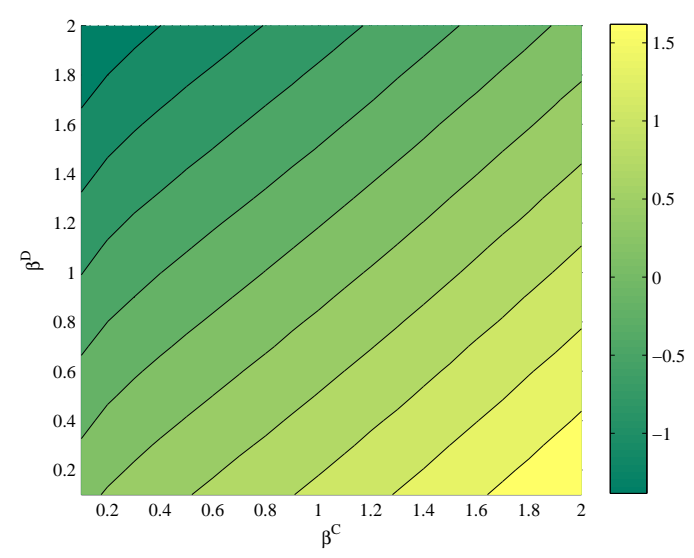

(d) Estimation error in $\hat{\beta}^{d}$ with 5-min data

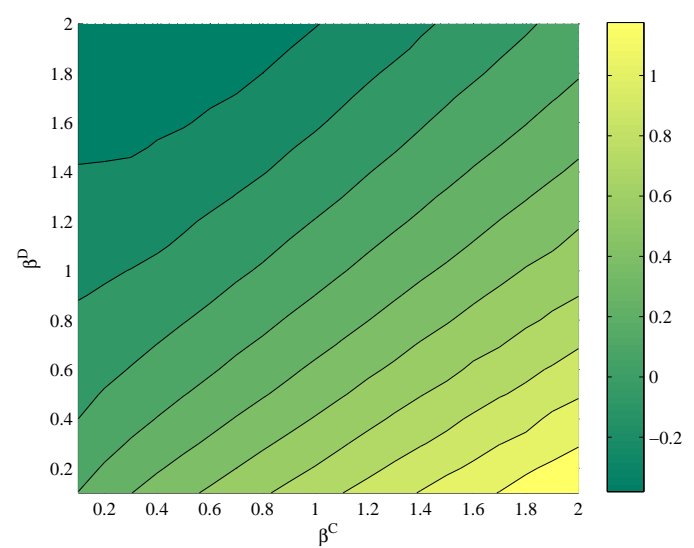

(f) Estimation error in $\hat{\beta}^{d}$ with 30 -sec data

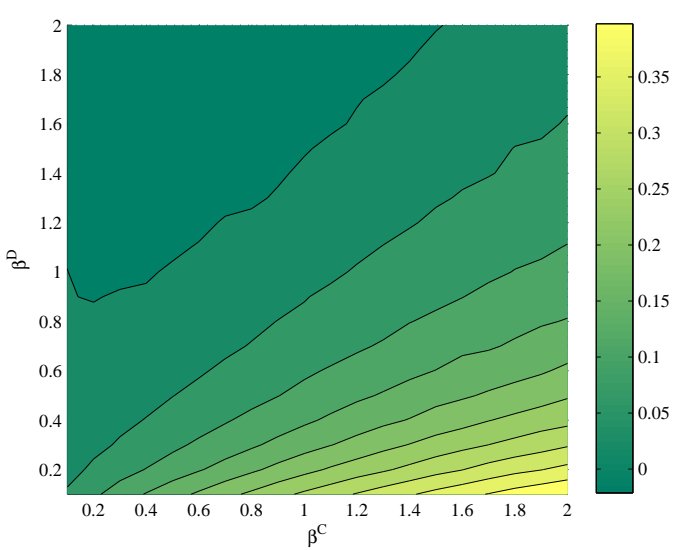


Figure 2: Estimation error in $\hat{\beta}^{c}$ and $\hat{\beta}^{d}$ against the difference between the true values of continuous and jump betas $\left(\beta^{c}-\beta^{d}\right)$ for 5-min data.

(a) Estimation error in $\hat{\beta}^{c}$

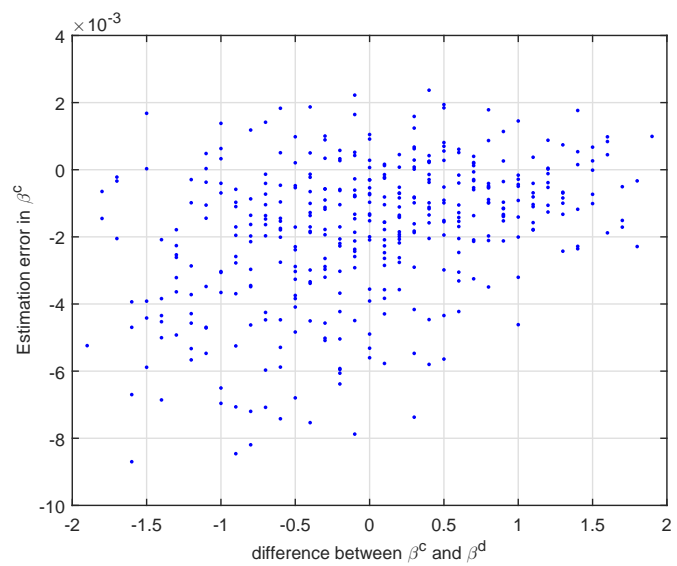

(b) Estimation error in $\hat{\beta}^{d}$

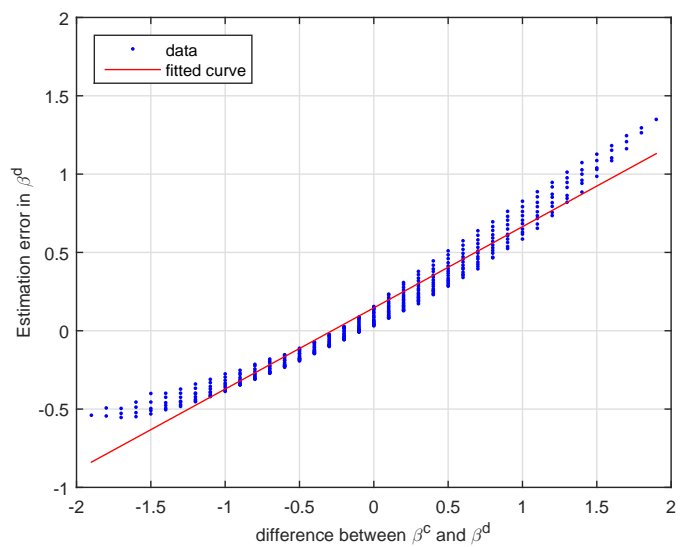

(a), the estimation error in $\hat{\beta}^{c}$ has no particular pattern and, overall, is trivially small. In contrast, Panel (b) of Figure 2 shows that when the difference between $\beta^{c}$ and $\beta^{d}$ is small the estimation error in $\hat{\beta}^{d}$ withers. Larger differences between $\beta^{c}$ and $\beta^{d}$, while exerting no impact on $\hat{\beta}^{c}$, lead to larger estimation errors in $\hat{\beta}^{d}$.

Understanding the finite sample properties of the estimators is important in how much reliability we can place on the implications of our results. Where the estimated difference between the continuous and jump betas is absolutely less than one, there is little evidence that we should be concerned. This turns out to be the case for our empirical example.

\section{Data and Parameter Choices}

We investigate the behavior of the $\beta_{i}^{c}$ and $\beta_{i}^{d}$ estimates over a 9 year sample period from January 2, 2003 to December 30, 2011, which includes the period of the financial crisis associated with the bankruptcy of Lehman Brothers in September 2008, and the subsequent period of turmoil in US and international financial markets. The underlying data are 5 minute observations on prices for 501 stocks drawn from the constituent stocks of the S\&P500 index during the sample period, obtained from SIRCA Thomson Reuters Tick History. This data set was constructed by Dungey et al. (2012) and does not purport to be all the stocks listed on the S\&P500 index, but has drawn from that population of stocks to select those with sufficient coverage and data availability for high frequency time series analysis of this type. 


\subsection{Data Processing}

The original data set consists of over 900 stocks taken from the 0\#.SPX mnemonic provided by SIRCA. This included a number of stocks which trade OTC and on alternative exchanges, as well as some which altered currency of trade during the period; these stocks were excluded. We adjusted the data set for changes in Reuters Identification Code (RIC) code during the sample period through mergers and acquisitions, stock splits and trading halts. We also removed some stocks with insufficient observations during the sample period. The data handling process is fully documented in the web-appendix to Dungey et al. (2012). In the data set for this paper, we force the inclusion of Lehman Brothers until their bankruptcy in September 2008, but drop Fannie Mae and Freddie Mac from the analysis. The final data set contains 501 individual stocks, hence $N=501$.

The intra-day returns and prices data start from 9:30 am, and end at 4:00 pm, observations with time stamps outside this window and overnight returns are removed. ${ }^{6}$ In the absence of trades within a 5-minute interval, prices are filled with the previous observation, corresponding to zero inter-interval returns. Approximately 20 price observations which are orders of magnitude away from their neighbouring observations are also removed. Thus we have 77 intra-day observations for 2262 active trading days belonging to 108 calendar months.

The 5 minute sampling frequency is chosen as relatively conventional in the high frequency literature, especially for univariate estimation, see for example Andersen et al. (2007), Lahaye et al. (2011), and for some sensitivity to alternatives Dungey et al. (2009). Optimal sampling frequency is an area of ongoing research, and despite the univariate work by Bandi and Russell (2006), this issue is outstanding for analysing multiple series with varying degrees of liquidity. The 5 minute frequency is much finer than those employed in Todorov and Bollerslev (2010) and Bollerslev et al. (2008) who use 22.5 minute data. Lower sampling frequencies are generally employed due to concerns over the Epps effect (Epps, 1979), however as the quality of high frequency data and market liquidity have improved in many ways, finer sampling does not threaten the robustness of our results. Patton and Verardo (2012) show the robustness of their results to 25 or 5 minute sampling, and we provide further evidence on sampling frequency in Appendix A and Appendix B.

Estimates of $\beta^{c}$ and $\beta^{d}$ are computed on a month-by-month basis. High frequency data permits the use of 1-month non-overlapping windows to analyse the dynamics of our systematic risk estimates. We also implement 12-month rolling windows to avoid interruptions in the discontinuous beta dynamics and to consider longer holding periods. For each month only liquid stocks are considered. Given the 5-minute sampling frequency, we define liquid stocks as ones that have at least $75 \%$ of the entire 1-month or 12 -month window as non-zero return data, which indicates that these stocks are heavily traded most of the time. We construct an equally weighted portfolio of all investible stocks in each estimation window as the benchmark market portfolio. We use equally weighted portfolios rather than value weighted ones to avoid situations where the weight on one stock is disproportionally large relative to other portfolio constituents.

\footnotetext{
${ }^{6}$ Sensitivity analysis to the inclusion of overnight returns showed no difference in the results, so we omit this in order to conserve space.
} 


\subsection{Choices of Parameter Values}

Although most of the parameters involved in the calculation of $\hat{\beta}^{\prime}$ s are nuisance parameters asymptotically, they do play important roles in any finite sample applications. We start the analysis by setting most of the parameter values to be the same as in Todorov and Bollerslev (2010), and then conduct robustness tests on the results obtained using different parameter settings.

Following the notation introduced earlier, there are $[T / \Delta]$ observations in $[0, T]$, thus $\Delta$ is set as the reciprocal of the number of observations in each month. The truncation threshold $\theta$ is chosen in accordance with Todorov and Bollerslev (2010) who set $\omega=0.49$, and let $a_{i}$ vary amongst individual stocks. Denote the bipower variation of the $i$-th stock over the time interval $[0, T]$ by $B V_{i}$, then we set $a_{i}=3 \sqrt{B V_{i}}, i=0,1, \ldots, N$.

Recall that when calculating $\hat{\beta}_{i}^{c}$ in equation (10), only those observations that satisfy $\left|\mathbf{r}_{j}\right| \leq \boldsymbol{\theta}$ are used. As the total number of stocks considered here, $N$, is quite high, this condition can be rather restrictive for all $N$ stock returns to be bounded below the threshold. We conduct sensitivity analysis and use different truncation thresholds to examine whether the estimated $\beta_{i}^{c}$ is robust to less (or more) restrictive truncation levels in Appendix C. Finally, the value of $\tau$ in equation (12) is set to $\tau=2$, consistent with Todorov and Bollerslev (2010).

\section{Properties of the Estimated Betas}

Using a significance level of $0.1 \%$, we detect jumps in our equally weighted market portfolio on 161 out of 2262 trading days using the statistic $\hat{\mathcal{J}}$ given in equation (16); that is $7.1 \%$ of all trading days. This corresponds to 82 jump months (months containing at least one jump day) out of the total 108 months in the sample. Patton and Verardo (2012) test the S\&P500 index between January 1996 and December 2006, a total of 2770 trading days, and find significant jumps on $4.04 \%$ of the days. Andersen et al. (2007), however, use the same ratio test statistic as applied here on the S\&P500 index from December 1986 through June 1999, and report 244 significant jump days (i.e. 7.6\%) out of 3045 trading days, at $0.1 \%$ significant level, which is consistent with our findings.

The estimated monthly $\hat{\beta}^{c}$ and $\hat{\beta}^{d}$ for individual stocks show that the estimated jump beta is higher than the continuous beta almost $90 \%$ of the time, indicating that most of the stocks are more sensitive to the sudden arrival of new information to the market than the generic market volatility. However during months of high market stress, such as September/December 2007, September/October 2008, November 2009 or May 2010, more than 30\% of the stocks have lower exposure to jump risk than the continuous risk. This result might seem counterintuitive, but in fact there are reasonable explanations for this phenomenon. First, beta measures the sensitivity of an individual stock to one unit of risk exposure. During crises the size of the jumps is generally larger, which means that when multiplied by a smaller beta coefficient in the crisis period it may still have an effect which may be higher than experienced in the non-crisis period where jumps are smaller (although the beta is larger). Second, crisis periods are characterised by increased market volatility. Thus, a given size of price discontinuity may be classified as a jump during a tranquil period but also reasonably fall in the category of a continuous price movement during a period of high volatility - 
shifting the impact from a jump beta to continuous beta estimate for that period. The reduction in jumps during periods of stress is consistent with the existing empirical results in Black et al. (2012), who report that the number of jumps during the crisis period is lower than the non-crisis period. The empirical application here finds evidence for only two market jump days from September to December 2007.

\subsection{Average Betas}

Figure 3 presents the means and medians of the estimated continuous and jump betas across the markets. ${ }^{7}$ The top panel plots means of the two beta estimates for each month, and the bottom panel plots medians.

Figure 3: Monthly $\hat{\beta}^{c}$ and $\hat{\beta}^{d}$ estimates. For each month, the top panel depicts the average $\hat{\beta}^{c}$ (solid line) across all investible S\&P500 stocks. The the average of $\hat{\beta}^{d}$ is plotted with asterisks. Similarly, the bottom panel depicts median values.
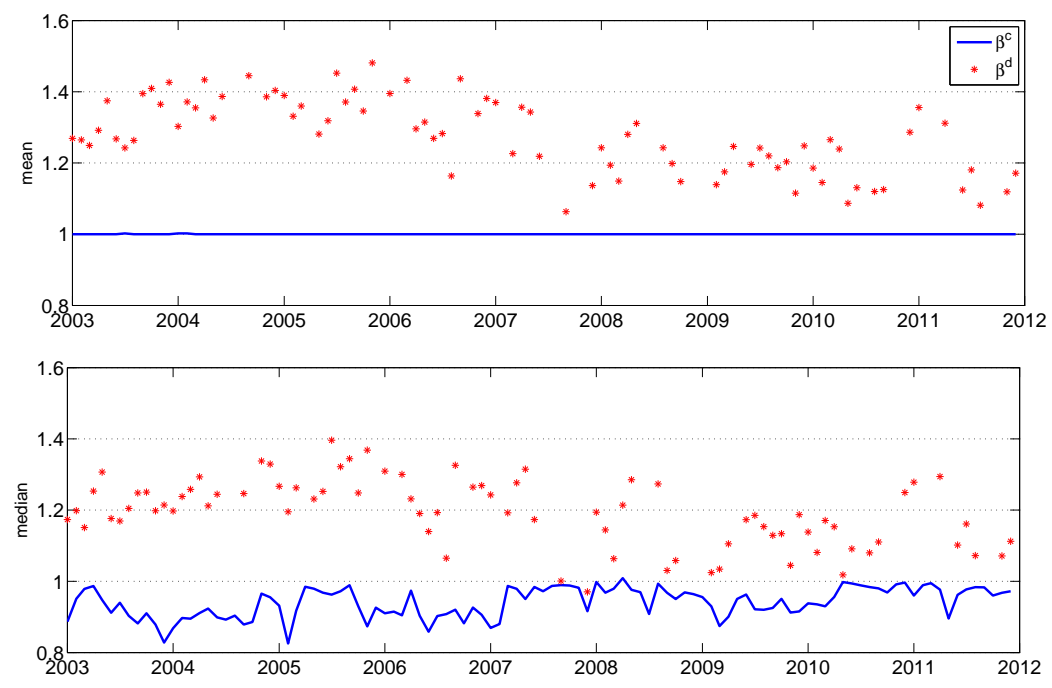

Since our market index is an equally weighted portfolio, by construction the mean of $\hat{\beta}^{c}$ is unity for every month. The jump betas are consistently higher than the continuous betas. ${ }^{8}$ The average impact of individual events (or news) which are sufficient to cause price discontinuities is largely contained in the range 1.1-1.5 (see Figure 3, top panel). The sample contains two periods of readily identifiable stress - the first in the third quarter of 2008 associated with the bankruptcy of Lehman Brothers, the rescue of AIG and the Trouble Assets Relief Program, and the second in the first half of 2010 associated with the Greek debt crisis - and in both of these periods the gap between the continuous and jump betas reduces. ${ }^{9}$ That is, there is more attention paid to volatility risk (the continuous component of the systematic risk) in the market than jump risk caused by news.

\footnotetext{
${ }^{7}$ Patton and Verardo (2012) compile results for estimated betas on event days across all stocks in the S\&P500 - that is a market wide estimate.

${ }^{8}$ Note that the mean of the estimated jump betas $\hat{\beta}^{d}$ is not necessarily unity, as the estimator given in equation (12) is not linearly addictive.

${ }^{9}$ Examples of identification of crisis period dates include Anand et al. (2013), Beber and Pagano (2013).
} 
For both the mean and median estimates, the gap between the discontinuous and continuous beta estimates has fallen over time. This can be partly explained by the high market volatility during the crisis periods. During times of high market stress, the overall market environment becomes relatively more important than unexpected news shocks to the system. The reaction to frequent unexpected news during stressed market times may be a feature of the overall market conditions.

\subsection{Firm Characteristics}

Firm characteristics can have strong impacts on firm sensitivity to systematic risk. For example, we would expect that larger firms are less vulnerable to market risks, and hence have lower beta compared with small firms. To explore the roles of firm characteristics in the estimates of $\hat{\beta}^{c}$ and $\hat{\beta}^{d}$, we rank stocks in terms of size, leverage, liquidity, level of idiosyncratic volatility, and longterm issuer credit risk rating in each month. We select the top and bottom $10 \%$ of stocks in our sample according to each characteristic, and compare the distributions of their beta estimates. Firm size is measured by market capitalization. The components of the leverage measure, book value of assets and equity, are available at daily frequency on Thomson Reuters Datastream. ${ }^{10}$ Liquidity is measured by the sum of cash and short term investments, divided by the book value of assets, which is only observed on a quarterly basis.

Following Bollerslev et al. (2016), we estimate the idiosyncratic volatility of each stock using a daily return regression:

$$
\bar{r}_{i, d}-\bar{r}_{f, d}=\alpha_{i}+\beta_{i}\left(\bar{r}_{0, d}-\bar{r}_{f, d}\right)+\phi S M B_{d}+\psi H M L_{d}+\epsilon_{i, d}
$$

where $\bar{r}_{i, d}$ and $\bar{r}_{0, d}$ are returns of the $i$-th stock and the market portfolio on day $d$, and $\bar{r}_{f, d}$ is the risk free rate on day $d . S M B_{d}$ and $H M L_{d}$ are the daily Fama-French factors downloaded from Kenneth French's website. Standard deviation of the regression residual in each month is taken as the idiosyncratic volatility of each stock.

We use the Standard \& Poor's Issuer Credit Rating (ICR) data, obtained through Wharton Research Data Services, to link the issuer's overall creditworthiness with the our high-frequency systematic risk estimates. Long-term ICR's range from AAA (extremely strong capacity to meet financial obligations) to CC (highly vulnerable). We constructed two categories of stocks - High Credit Ranking category consisting of prime and high grade rated issuers (AAA, AA+, AA, and AA- rankings) and Low Credit Ranking category consisting of highly speculative and extremely speculative issuers (B+, B, B-, CCC+, CCC, CCC-, and CC rankings). ${ }^{11}$

Figures 4-7 plot the median, inter-quartile range, minimum and maximum values for $\hat{\beta}^{c}$ and $\hat{\beta}^{d}$ of sorted top and bottom securities, respectively. As expected, larger firms usually have lower exposure to both the continuous and jump components of the systematic risk. For the largest $10 \%$ of firms in the data set, the two beta estimates both display a decreasing trend over the entire sample

\footnotetext{
${ }^{10}$ Leverage is measured in log levels scaled by the standard deviation of leverage for all firms in the sample.

${ }^{11}$ Rankings C (default imminent with little prospect for recovery) and D (in default) were not observed in the Standard \& Poor's Issuer Credit Rating (ICR) data despite that out sample of stocks included Lehman Brothers Holdings Inc.
} 
Figure 4: Comparison of $\hat{\beta}^{c}$ according to firm characteristics
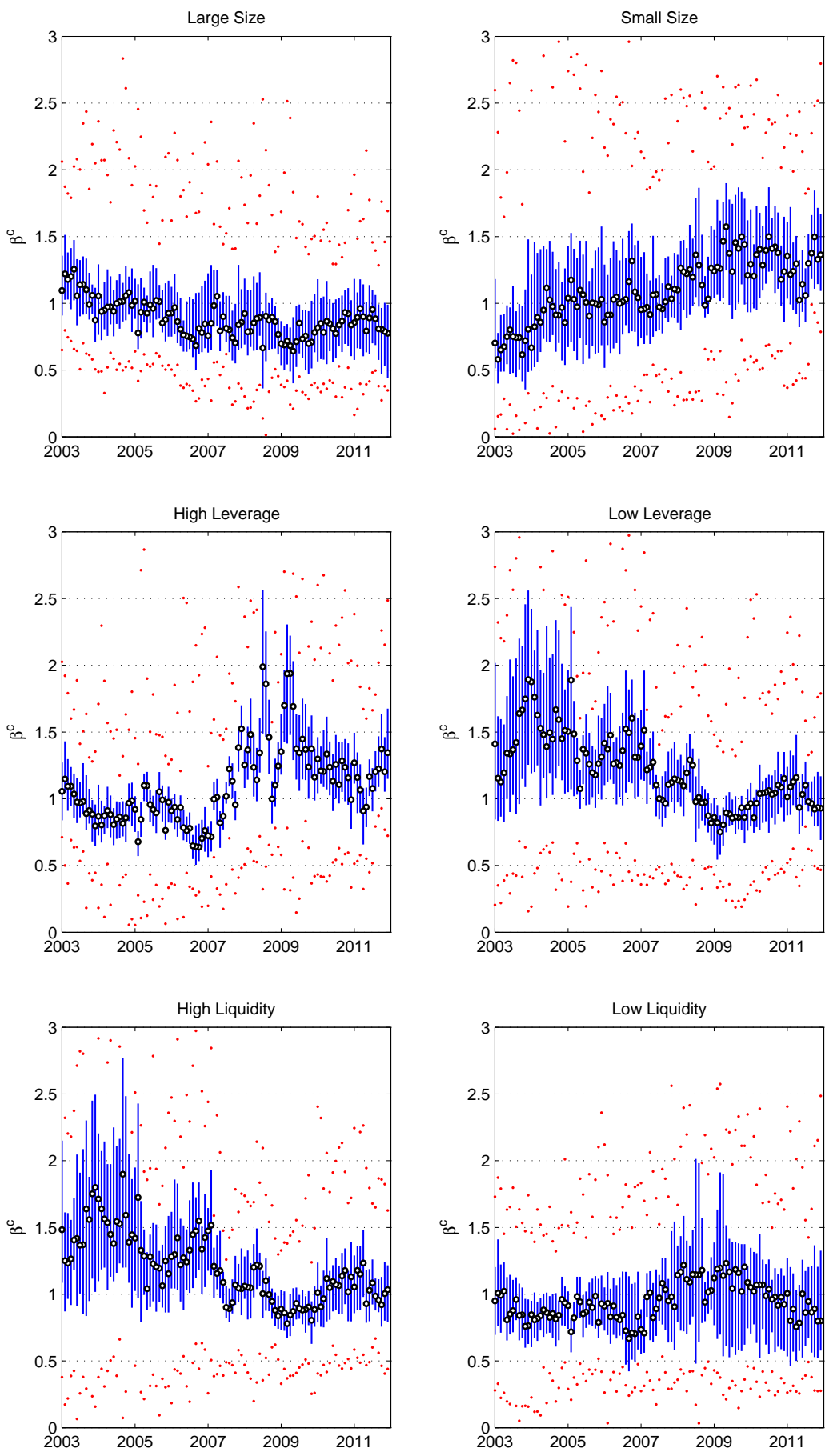

The black circles denote the median value of the estimated $\beta^{c}$ in each month, the blue vertical lines denote the inter-quartile range, and the red dots denote the minimum and maximum values for each group. 
Figure 5: Comparison of $\hat{\beta}^{c}$ according to firm characteristics
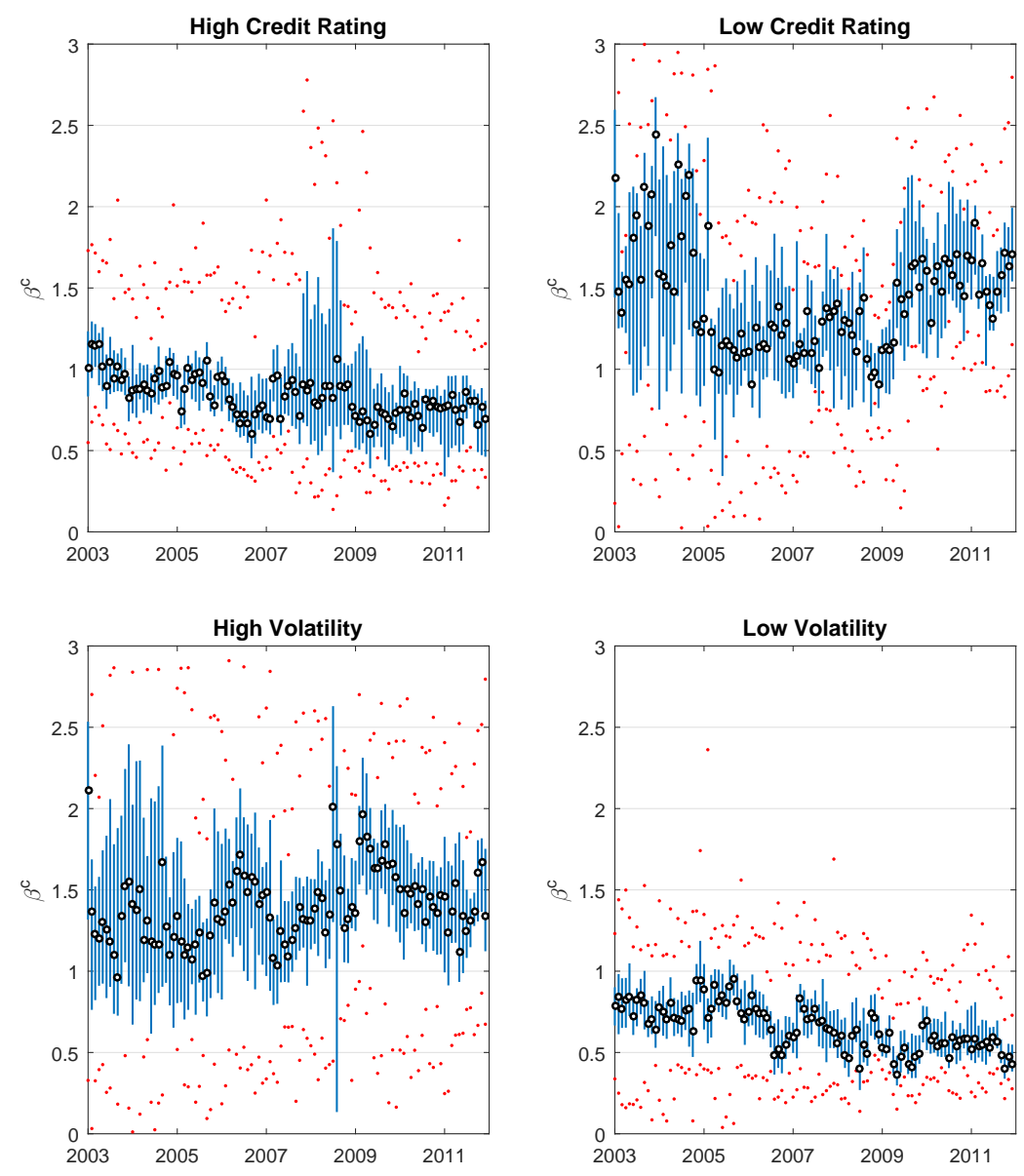

The black circles denote the median value of the estimated $\beta^{c}$ in each month, the blue vertical lines denote the inter-quartile range, and the red dots denote the minimum and maximum values for each group. 
Figure 6: Comparison of $\hat{\beta}^{d}$ according to firm characteristics
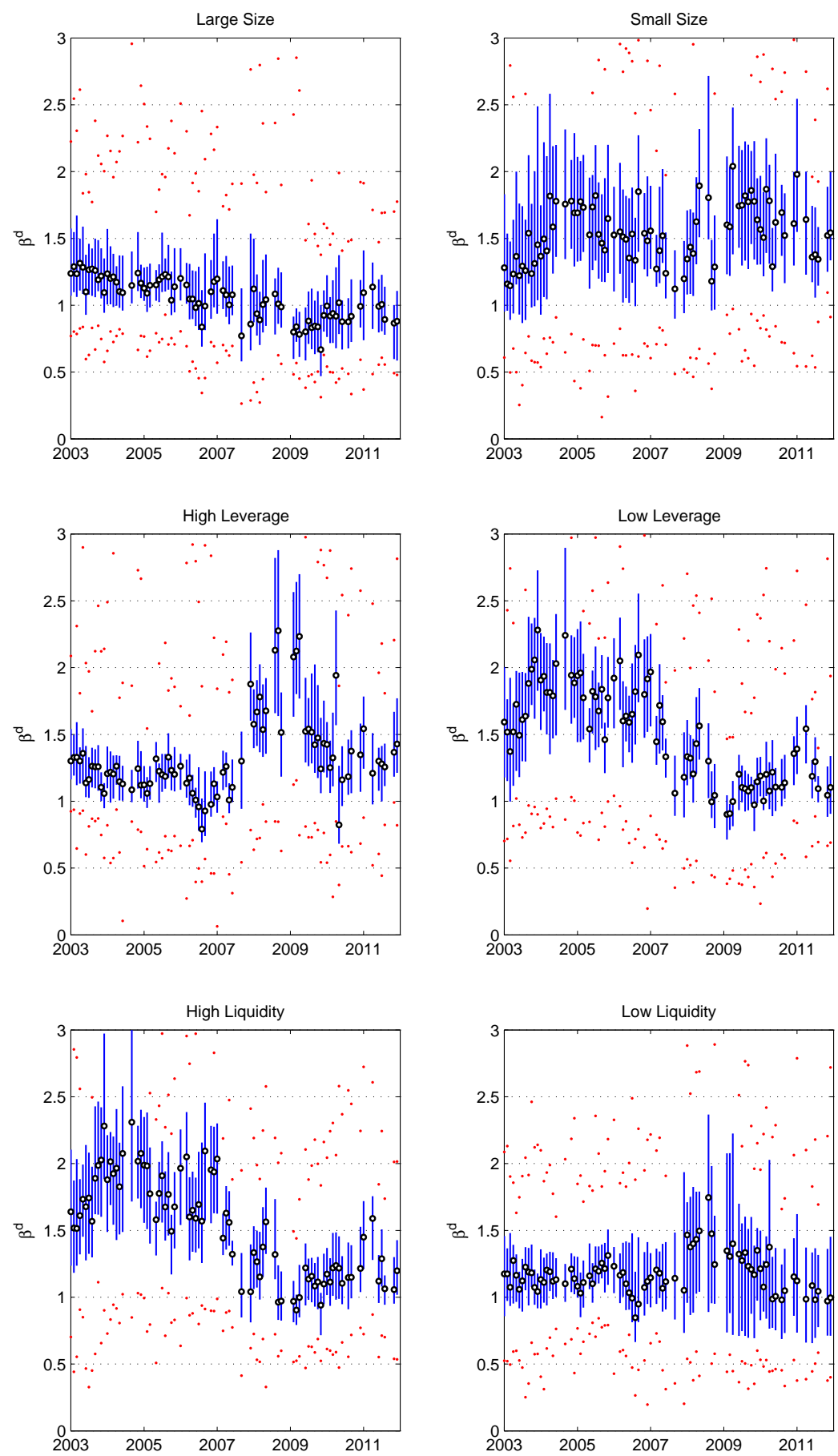

The black circles denote the median value of the estimated $\beta^{d}$ in each month, the blue vertical lines denote the inter-quartile range, and the red dots denote the minimum and maximum values for each group. 
Figure 7: Comparison of $\hat{\beta}^{d}$ according to firm characteristics
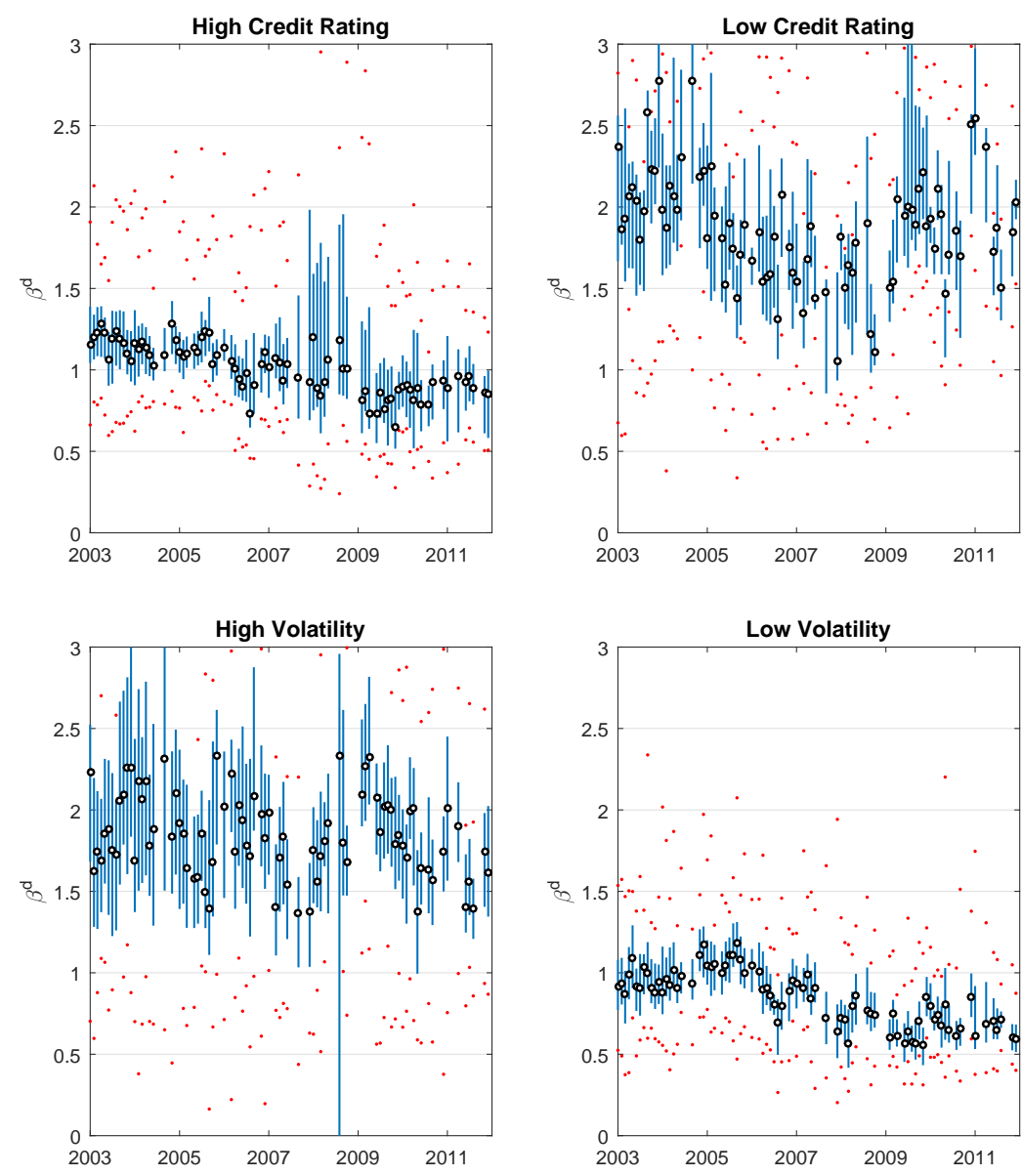

The black circles denote the median value of the estimated $\beta^{c}$ in each month, the blue vertical lines denote the inter-quartile range, and the red dots denote the minimum and maximum values for each group. 
period. In contrast, the smallest firms experience higher exposure to the continuous systematic risk as time goes by. Higher market volatility resulting from the crisis exaggerates the overall effect even further. The difference between the largest and the smallest firms is more profound for jump risk than continuous risk.

Figures 4 and 6 provide evidence that firms with the highest and lowest levels of leverage (or liquidity) exhibit distinctive patterns in the two beta estimates, with what appears to be a structural shift during late 2007 to 2008 when the crisis begins. During the crisis high leverage stocks are more exposed (with higher continuous and jump betas) while high liquidity stocks become less exposed to systematic risk (lower betas) during the crisis period. Firms with larger debt obligations (relative to equity) are more sensitive to market fluctuations during financial distress. It is not surprising that firms with low debt are seen as attractive during volatile times and become safe havens for investors. "Cash cows" or stocks with high levels of liquidity are considered more defensive and have reduced sensitivity during turbulent market times. ${ }^{12}$

The top panels in Figures 5 and 7 show that both continuous and discontinuous betas for stocks with high credit ratings are strikingly lower than the corresponding betas for stocks with low credit ratings, and with a much wider inter-quartile range. The only exception is the period of the GFC where the differences in beta magnitudes between the two credit rating categories is less pronounced. In many instances for the stocks with low idiosyncratic volatility, both the continuous and discontinuous betas are below one. Higher volatility is commonly associated with higher values of betas, consistent with the findings in Bollerslev et al. (2016).

\subsection{Industry Characteristics}

We analyze the industry composition of the largest and smallest $10 \%$ of continuous and discontinuous beta estimates, over our full sample and two sub-samples representing pre-crisis and crisis periods. We choose July 2007 as the breakpoint in the sample, consistent with the emergence of the first problems with Bear-Stearns. This coincidentally splits the sample into two equally sized subsamples of 54 months each. The mean and median of the highest $10 \%$ of $\hat{\beta}^{c}$ vary within $(1.4,2.5)$ throughout the sample period, whereas the mean and median of the lowest $10 \%$ of $\hat{\beta}^{c}$ stay within $(0.04,0.63)$. The estimated discontinuous betas are more volatile, where the mean and median of the highest $10 \%$ lie in $(1.5,3.2)$, and the mean and median of the lowest $10 \%$ fall in $(-0.75,0.9)$ over the full sample.

Table 1 breaks down the industry classifications of firms present in the highest $10 \%$ of the estimated $\hat{\beta}_{i}^{c}$ and $\hat{\beta}_{i}^{d}$, as a percentage of the total firms in the sample (recall that the composition of the index varies with time). The first column of the table gives the percentage of firms by industry in the S\&P500 index. The following columns provide the percentage of firms belonging to each industry for the different sample periods based on the estimated continuous and jump betas. Panel A (the

\footnotetext{
${ }^{12}$ New Cash Cows: Biggest Stocks, The Wall Street Journal (Jan 6, 2006) names a number of companies with increasing cash: Citigroup, H.J. Heinz, Pfizer, Sara Lee, Merck, BofA, Verizon Communications, and the following five dividend cash cows - IBM, Chevron, AT\&T, 3M and PepsiCo.
} 
Table 1: Classification of large systematic impacts by industry (all values are in percentage terms)

\begin{tabular}{|c|c|c|c|c|c|c|c|}
\hline \multirow[t]{2}{*}{ Industry ${ }^{\mathrm{a}}$} & \multirow[t]{2}{*}{$\begin{array}{c}\text { S\&P500 } \\
\text { represen- } \\
\text { tation }^{b}\end{array}$} & \multicolumn{2}{|c|}{$\begin{array}{c}\text { Full period } \\
(2003: 01) \\
(-2011: 12)\end{array}$} & \multicolumn{2}{|c|}{$\begin{array}{c}\text { Pre-crisis } \\
(2003: 01) \\
(-2007: 06)\end{array}$} & \multicolumn{2}{|c|}{$\begin{array}{c}\text { Crisis } \\
(2007: 07) \\
(-2011: 12)\end{array}$} \\
\hline & & $\hat{\beta}_{i}^{c}$ & $\hat{\beta}_{i}^{d}$ & $\hat{\beta}_{i}^{c}$ & $\hat{\beta}_{i}^{d}$ & $\overline{\hat{\beta}_{i}^{c}}$ & $\hat{\beta}_{i}^{d}$ \\
\hline \multicolumn{8}{|c|}{ Panel A: Breakdown of the largest $10 \%$ of $\hat{\beta}^{\prime}$ s (most sensitive stocks) } \\
\hline Basic Materials & 12.7 & 26.0 & 15.9 & 24.2 & 15.4 & 27.7 & 15.7 \\
\hline Conglomerates & 1.5 & 0.3 & 0.1 & 0.1 & 0.0 & 0.6 & 0.5 \\
\hline Consumer Goods & 11.1 & 3.6 & 4.2 & 1.5 & 2.2 & 5.9 & 8.2 \\
\hline Financial & 15.2 & 16.1 & 13.0 & 1.5 & 1.6 & 32.0 & 33.4 \\
\hline Healthcare & 9.2 & 1.6 & 3.1 & 2.8 & 3.9 & 0.1 & 0.4 \\
\hline Industrial Goods & 10.4 & 10.5 & 11.7 & 6.9 & 9.8 & 14.0 & 14.9 \\
\hline Services & 19.3 & 10.9 & 16.9 & 11.5 & 16.7 & 10.1 & 16.2 \\
\hline Technology & 14.9 & 30.9 & 34.4 & 51.1 & 49.7 & 9.6 & 10.3 \\
\hline Utilities & 5.9 & 0.2 & 0.6 & 0.4 & 0.6 & 0.0 & 0.3 \\
\hline \multicolumn{8}{|c|}{ Panel B: Breakdown of the smallest $10 \%$ of $\hat{\beta}^{\prime}$ s (most defensive stocks) } \\
\hline Basic Materials & 12.7 & 7.4 & 6.2 & 11.3 & 9.7 & 3.8 & 5.5 \\
\hline Conglomerates & 1.5 & 0.4 & 0.4 & 0.9 & 0.7 & 0.0 & 0.2 \\
\hline Consumer Goods & 11.1 & 24.5 & 24.7 & 16.7 & 21.1 & 31.9 & 31.5 \\
\hline Financial & 15.2 & 10.5 & 8.0 & 20.4 & 19.0 & 1.2 & 2.5 \\
\hline Healthcare & 9.2 & 20.3 & 20.9 & 12.2 & 13.7 & 28.1 & 24.7 \\
\hline Industrial Goods & 10.4 & 2.9 & 3.3 & 4.3 & 3.8 & 1.5 & 1.7 \\
\hline Services & 19.3 & 12.7 & 12.7 & 13.7 & 13.1 & 10.6 & 11.0 \\
\hline Technology & 14.9 & 6.3 & 7.2 & 8.5 & 4.5 & 4.3 & 5.6 \\
\hline Utilities & 5.9 & 15.5 & 16.7 & 12.1 & 13.9 & 18.6 & 17.3 \\
\hline
\end{tabular}

${ }^{a}$ We analyze the industry effect by concentrating on the largest impacts of the two systematic components. Relative representation of each industry in the sample is found by (i) accounting for existence of each of the firm in the sample during each of the month over the entire period from 2003 to 2011; (ii) its inclusion in selected industry; and (iii) for $\hat{\beta}_{i}^{d \prime}$ 's only, the existence of a jump day in a month.

${ }^{b}$ Industry breakdown of all of the S\&P500 constituents.

top panel) provides the results for the most sensitive stocks, those with the top $10 \%$ of estimated $\hat{\beta}_{i}^{c}$ and $\hat{\beta}_{i}^{d}$. Panel B (the lower panel) provides the results for the least sensitive $10 \%$ of the stocks.

The most obvious feature in the sectoral analysis concerns the changing role of Financial sector stocks across the pre-crisis and crisis periods. Financial sector stocks comprise only $1.5 \%$ of the most sensitive stocks in the pre-crisis period, and are the most plentifully represented amongst the most defensive stocks at $20.4 \%$. In sharp contrast, Financial stocks account for $32.0 \%$ of the most sensitive stocks and $1.2 \%$ of the least sensitive stocks in the crisis half of the sample. This reflects the central role of the financial sector in the crisis, and is consistent with very high systematic risk during the period of extreme financial stress.

In the pre-crisis period the Technology sector dominates the set of the most sensitive stocks with more than half of the most sensitive stocks from this sector for both continuous and jump systematic risks. This is not surprising given the aftermath of the burst of the Dot-com bubble a few years 
earlier. From mid-2007, as stress builds in the Financial sector the Technology sector becomes much less sensitive to risks in the market portfolio, reflecting its relative independence from other sectors of the economy - consistent with the industry boom and burst cycles evident in specific competitive industries in Hoberg and Phillips (2010).

In both the pre-crisis and crisis periods the second most common sector for firms with high exposure to continuous systematic risk is Basic Materials. While the proportion of firms from the Basic Materials sector is also relatively high for jump beta, firms from the Service sector are slightly more prevalent. We do not observe the same phenomenon among defensive stocks in the Basic Materials sector.

The Consumer Goods sector is the most consistently found amongst the defensive stocks in the full sample, and represents $31.9 \%$ of the stocks in the crisis period for continuous beta. This sector is negligible in the most sensitive stocks of panel $\mathrm{A}$, although it represents $8.2 \%$ of stocks classified according to most responsive jump betas. Healthcare is also relatively unrepresented in the more sensitive stocks, but accounts for $28 \%$ (almost $25 \%$ ) of the most defensive stocks in the crisis period using continuous (jump) betas - around double its representation in defensive stocks during the pre-crisis sample.

In summary, the leading sectors among the most sensitive (or aggressive) stocks are: Technology (only in the pre-crisis period), Financial (only in the crisis period), Basic Materials and Services. Healthcare, Consumer Goods and the Financial sector (only in pre-crisis period) prevail among the most defensive stocks.

\section{Risk Premia Decomposition}

According to equation (2), overall systematic risk can be decomposed into two distinct components - continuous risk and jump risk, where the factor loadings, $\beta_{i}^{c}$ and $\beta_{i}^{d}$ measure the sensitivities of individual stocks towards the two risk factors. Having these two distinct beta estimates for all 501 constituent stocks allows us to estimate risk premia on each of the systematic risk components.

The approach implemented in Todorov and Bollerslev (2010) does not allow for a direct breakdown of market returns themselves into continuous and discontinuous components. However, viewing these two components as separate risk factors to firms that are exposed to market fluctuations suggests that the two-stage Fama and MacBeth (1973) regression can be used to estimate premia rewarded to each of these factors. ${ }^{13}$ As these two factors drive stock returns, this approach can be used to price how much return one would expect to receive for a particular level of systematic factor exposure.

As a first stage we take the estimates of $\hat{\beta}_{i}^{c}$ and $\hat{\beta}_{i}^{d}$ from the model in (2) for each asset, $i=$

\footnotetext{
${ }^{13}$ Despite identified errors-in-variables problem, cross-sectional dependence and the Roll Critique (Roll, 1977; Roll and Ross, 1994), Fama and MacBeth (1973) remains by far the most popular method in the empirical literature when estimating risk premia. Numerous improvements to correct for serial correlation, heteroscedasticity and errors-in-variables have been suggested dating a few decades as well as recently with no uniform widely accepted parsimonious model (see, e.g. Ferson and Harvey, 1991; Giordani and Kohn, 2008).
} 
$1, \ldots, N$, and each time period (each month in our case). These reveal the extent to which each asset return is influenced by the continuous and discontinuous movements in the market as discussed in the previous section.

In order to extract the premia we undertake a second stage consisting of a set of $S$ regressions; we estimate risk premia using betas computed (i) monthly, resulting in $S=108$ distinct estimates; and (ii) annually, with 12-month rolling windows for each month resulting in $S=97$ distinct estimates. We run cross-sectional regressions for each month $s=1, \ldots, S$ in the following form

$$
\bar{r}_{i, s}=\alpha_{s}+\gamma_{s}^{c} \hat{\beta}_{i, s-1}^{c}+\gamma_{s}^{d} \hat{\beta}_{i, s-1}^{d}+\epsilon_{i, s}
$$

where $\bar{r}_{i, s}$ is the average monthly return on stock $i$ in the $s$-th month and $\hat{\beta}_{i, s-1}^{c}$ and $\hat{\beta}_{i, s-1}^{d}$ are the two systematic risk factors estimated from the previous month. To calculate the average monthly risk premia we take the sample mean of the estimates obtained from (18):

$$
\bar{\gamma}^{c}=\frac{1}{S} \sum_{s=1}^{S} \hat{\gamma}_{s}^{c}, \quad \text { and } \quad \bar{\gamma}^{d}=\frac{1}{S} \sum_{s=1}^{S} \hat{\gamma}_{s}^{d}
$$

The average coefficients in (19) represent the premia awarded for one unit of factor exposure of continuous and discontinuous market fluctuations, respectively.

Table 2 shows the average estimated coefficients in equation (19), $\bar{\gamma}^{c}$ and $\bar{\gamma}^{d}$ for the entire sample period from 2003:01 to 2011:12 as well as for the pre-crisis (2003:01-2007:06) and crisis (2007:072011:12) periods ${ }^{14}$; significance levels are assessed with Newey-West corrected $t$-statistics. The two sub-columns for $\bar{\gamma}^{c}$ and $\bar{\gamma}^{d}$ in Table 2 denote the two different beta estimates: (i) betas are estimated using last month's data prior to the average monthly return; (ii) betas are estimated using 12-month rolling window prior to the current month of the average monthly return. Table 2 suggests that the results from these two different model specifications are qualitatively similar. During the precrisis period, we observe significantly negative premia for the continuous risk factor, while the risk premia for the discontinuous component is significantly positive. However, when we isolate the crisis period only, neither of the two risk premia are significant. We hypothesize that this is due to increased volatility in average monthly returns of the assets during the crisis, whereas the beta estimates do not appear to exhibit high fluctuations.

The finding that discontinuous risk receives significantly positive premium but continuous risk does not, is consistent with the conclusion contemporaneously drawn with cross-sectional data by Bollerslev et al. (2016) over the period 1993 to 2010. However, sub-sample analysis reported in the bottom panel of Table 2 compares the estimated risk premia for the pre-crisis period and the crisis period, with the result that in the pre-crisis period both continuous and discontinuous risk premia are significant, while during the crisis both premia are insignificant, leaving the market unable to distinguish whether it is more concerned about continuous or discontinuous systematic risk during the crisis period.

${ }^{14}$ The median values for these coefficients are very similar. 
Table 2: Average risk premia for individual sectors using Fama-MacBeth estimation

\begin{tabular}{|c|c|c|c|c|}
\hline & \multicolumn{2}{|c|}{ Continuous risk premia $\left(\bar{\gamma}^{c}\right)$} & \multicolumn{2}{|c|}{ Discontinuous risk premia $\left(\bar{\gamma}^{d}\right)$} \\
\hline & (i) & (ii) & (i) & (ii) \\
\hline \multicolumn{5}{|l|}{ 5-min sample } \\
\hline Full period & -0.0073 & -0.0012 & 0.0069 & -0.0052 \\
\hline Pre-crisis & $-0.0083^{*}$ & $-0.0202^{* *}$ & 0.0093 & $0.0169^{* *}$ \\
\hline Crisis & -0.0061 & 0.0136 & 0.0039 & -0.0223 \\
\hline \multicolumn{5}{|c|}{ 15-min sample } \\
\hline Full period & -0.0038 & -0.0031 & 0.0099 & 0.0010 \\
\hline Pre-crisis & $-0.0064^{*}$ & $-0.0222^{* *}$ & 0.0046 & $0.0203^{* *}$ \\
\hline Crisis & -0.0012 & 0.0118 & 0.0152 & -0.0201 \\
\hline \multicolumn{5}{|c|}{ 30-min sample } \\
\hline Full period & 0.0027 & -0.0010 & -0.0018 & -0.0027 \\
\hline Pre-crisis & $-0.0053^{*}$ & $-0.0220^{* *}$ & $0.0092^{*}$ & $0.0213^{* *}$ \\
\hline Crisis & 0.0108 & 0.0152 & -0.0131 & -0.0213 \\
\hline \multicolumn{5}{|c|}{ 75-min sample } \\
\hline Full period & -0.0004 & 0.0013 & -0.0018 & -0.0052 \\
\hline Pre-crisis & 0.0011 & -0.0082 & 0.0040 & $0.0085^{+}$ \\
\hline Crisis & -0.0019 & 0.0087 & -0.0075 & -0.0159 \\
\hline
\end{tabular}

a Numbers denote the risk premia awarded for one unit factor exposure to continuous and discontinuous market fluctuations.

${ }^{b}$ Two model specifications used to estimate the continuous and jump betas are: (i) one period lagged 1month non-overlapping window; (ii) one period lagged 12-month rolling window. All regressions include the intercept term.

c Significance levels: $\quad+: 10 \%, \quad *: 5 \%, \quad * *: 1 \%$. 
Table 2 also demonstrates the robustness of the Fama-MacBeth risk premia estimation with respect to different sampling frequency. Using 15-min, 30-min, and 75-min sampling frequencies, we continue to obtain positive and significant risk premia for discontinuous risk in pre-crisis period. The risk premia for the continuous beta is usually negative. During crisis period, neither the continuous nor the discontinuous risks carries significant premia. It is clear that the general findings based on the 5-min sampling frequency shown in the top panel of Table 2 are preserved when more sparse sampling schemes are used.

Using the results in Table 2 for our preferred model (ii), a 2-standard-deviation difference in $\beta^{d}$ during pre-crisis period, for the 5-min sampling frequency will lead to a difference in expected return of $2 \times 0.4287 \times 0.0169 \times 12=0.1739$ or $(17.39 \%)$ per year, which is economically large difference in expected return. Using 75-min frequency, the difference in expected return associated with 2 standard deviation in $\beta^{d}$ during pre-crisis period is $2 \times 0.4826 \times 0.0085 \times 12=0.0985$ or $(9.85 \%)$ per year. This is very close to the estimates in Bollerslev et al. (2016).

The reliability of our results may be obfuscated by the presence of financially distressed firms, whose stocks usually exhibit low returns, yet have high betas. Campbell et al. (2008) argue that this pattern is inconsistent with the conjecture that the value and size effects are compensation for the risk of financial distress. The proportion of financially distressed stocks may be larger in the crisis period further diluting the results. Chan and Chen (1991, p. 1468) discuss the prevalence of financially distressed stocks in small-stock portfolios and characterise them as highly leveraged, inefficient firms with cash flow problems. As previously discussed the betas for the most leveraged, largest and smallest firms show distinct patterns in the estimated continuous and jump betas reported in Figures 4 and 6. To check whether avoiding small-sized firms results in a clearer relationship between returns and the estimated betas we perform the analysis discussed in this section with only largest 100 firms, and obtain very similar results.

\section{Conclusion}

The recent literature in high-frequency financial econometrics has provided evidence that price discontinuities, or jumps, are important features of the price process. With the advent of techniques to separate jumps from the continuous component of the price process, we can now distinguish between the continuous and the jump systematic risk components in the market portfolio and capture the time variation in those estimated betas over relatively short intervals.

This paper estimates the CAPM beta for both continuous and jump components for constituent stocks of the S\&P500 index over the sample period of 2003 to 2011 using a new 5 minute data set compiled from Thompson Reuters Tick History. We find that on average the jump betas are usually $30 \%-40 \%$ higher than the continuous betas. These estimates suggest that when news is sufficient to disrupt prices, that is to cause a jump, the speed with which news is disseminated into the market is likely to be even faster than previously estimated using the combined continuous and jump price process as in Patton and Verardo (2012). 
The industry breakdown for both the continuous and jump betas show distinct changes between pre-crisis and crisis periods. Most industries experience substantial changes in their sensitivities to the continuous systematic risk associated with the global financial crisis. Not surprisingly, the Financial sector displays the most volatility in the beta estimates in this period, especially during the pre-crisis period, when the Financial sector was considered defensive. For most of the stocks, the only time that the jump beta is not higher than the continuous beta is during periods of high financial market distress - in late 2008 and the first half of 2010 due to the emerging Greek crisis. In these periods systematic risk considerations dominate any concerns about individual news events. Overall we find that the leading sectors among the most aggressive stocks are: Technology (only in the pre-crisis period), Financial (only in the crisis period), Basic Materials and Services. Healthcare, Consumer Goods and Financial (only in pre-crisis period) prevail among the most defensive stocks.

In addition to the breakdown of the highest and lowest beta estimates across different industries, we analyse the relationship between firm characteristics and beta estimates. We find that smaller stocks are more sensitive to jumps than their larger counterparts. The differences between continuous and discontinuous betas are more evident for smaller stocks. During periods of financial market distress, high leverage stocks are more exposed to unexpected market-wide news. High liquidity stocks are found to be more aggressive during pre-crisis period. However during the crisis period, these stocks are among those which have the lowest sensitivities to market fluctuations (defensive stocks). Stocks with low credit ratings have higher continuous and discontinuous betas than highly rated stocks. Stocks with high idiosyncratic volatility have both higher and more volatile continuous and discontinuous betas than their low idiosyncratic volatility counterparts. We find evidence of positive risk premia for the discontinuous risk, and negative premia for the continuous risk, although the statistical significance of both these premia disappears during the crisis period.

Our results show that systematic risk factors differ in periods of high market volatility from those in low volatility periods. Future research will investigate asymmetric effects in beta in response to positive and negative jumps and news. This requires further analysis of the properties of the Todorov and Bollerslev (2010) tests to accommodate the detection of the direction, as well as the existence, of jumps.

\section{Appendix A: Epps Effect}

Epps (1979) first documented that the correlations among stock returns decrease to zero when their sampling intervals decrease. In the context of the present paper, the Epps effect will cause downward bias in the estimated betas, and is more pronounced for non-liquid stocks. This is the main reason that Todorov and Bollerslev (2010) and Patton and Verardo (2012) choose to use relatively low sampling frequencies in their beta estimates. In order to ensure that our results are not driven by the Epps effect, we construct price and return series at 10, 20 and 30 minutes sampling intervals, and re-calculate $\hat{\beta}_{i}^{c}$ and $\hat{\beta}_{i}^{d}$ for all 501 stocks in the dataset. Figure A.8 plots the monthly 
$\hat{\beta}_{i}^{c}$ for the four smallest firms in terms of Size, as they are less liquid and more prone to the Epps effect. Figure A.8 reveals that the original estimates $\left(\beta^{c}(5 \mathrm{~min})\right)$ are less volatile but not necessarily smaller than the $\hat{\beta}_{i}^{c}$ calculated using 20-minutes and 30-minutes returns. They exhibit a qualitatively similar pattern over the entire sample period. The same finding still holds for other stocks in this S\&P500 dataset. These results are available upon request.

\section{Appendix B: Beta signature plots}

Figure B.9 depicts continuous and discontinuous beta signature plots for an exemplar of 10 stocks, where we included both large and small capitalisation companies. ${ }^{15}$ We use 5-min, 15min, 30-min, and 75-min sampling frequencies to estimate betas for each month. For each sampling frequency, panels (a) and (b) in Figure B.9 depict the average monthly continuous and discontinuous betas for each of the 10 stocks, respectively. We do not observe substantial changes in our beta estimates. This is in contrast to Figure 3 Panel A in Bollerslev et al. (2016, p.482), where the authors show that the continuous beta estimates suffer from microstructure noise at higher frequencies. Our results for the estimates of continuous beta are stable across all frequencies since our sample of stocks focuses on the largest 500 stocks on the US market with an average of 420 stocks per month, while the sample in Bollerslev et al. (2016) consists of the 985 stocks with 738 stocks per month on average. The difference in these two samples could be due to relatively less liquid stocks with smaller capitalisations prone to higher microstructure noise.

\section{Appendix C: Robustness Test}

Here we investigate the robustness of the empirical results obtained in previous analysis with respect to different choices of the parameter values. Most of these nuisance parameters do not affect the consistency property of the estimators, but will impact finite sample applications.

For monthly estimates of the $\beta^{\prime}$ s, the value of $\Delta$ is changing from one month to another, because the number of observations are different. Thus we choose an average number of observations per month to set $\Delta=1 / 1613$ for all calender months, and calculate the monthly $\hat{\beta}^{c}$ and $\hat{\beta}^{d}$ for all stocks. There are still 82 months that contain at least one jump day using the adjusted ratio test by Barndorff-Nielsen and Shephard (2006). Both $\hat{\beta}^{c}$ and $\hat{\beta}^{d}$ are almost the same as our previous results.

Next we consider different values for $\alpha_{i}$ in the truncation threshold $\theta$ in (11). Changing the value of $a_{i}$ will affect the number of observations involved in the calculation of $\hat{\beta}_{i}^{c}$, hence we cannot set $a_{i}$ too restrictively in order to retain sufficient observations to estimate (10). Notice that in equation (10), $\left|r_{i, j}\right| \leq a_{i} \Delta^{\omega}$ needs to be satisfied for each $i=0,1, \ldots, N$. For large $N$ we can lose a considerable number of observations using this truncation threshold. Thus we only examine the

\footnotetext{
${ }^{15}$ We estimate beta signature plots for all 501 stocks in our sample, but present only a representative subset for brevity. Full set of results is available upon request.
} 
Figure A.8: The monthly $\hat{\beta}^{c}$ estimated under different sampling frequencies for small size firms
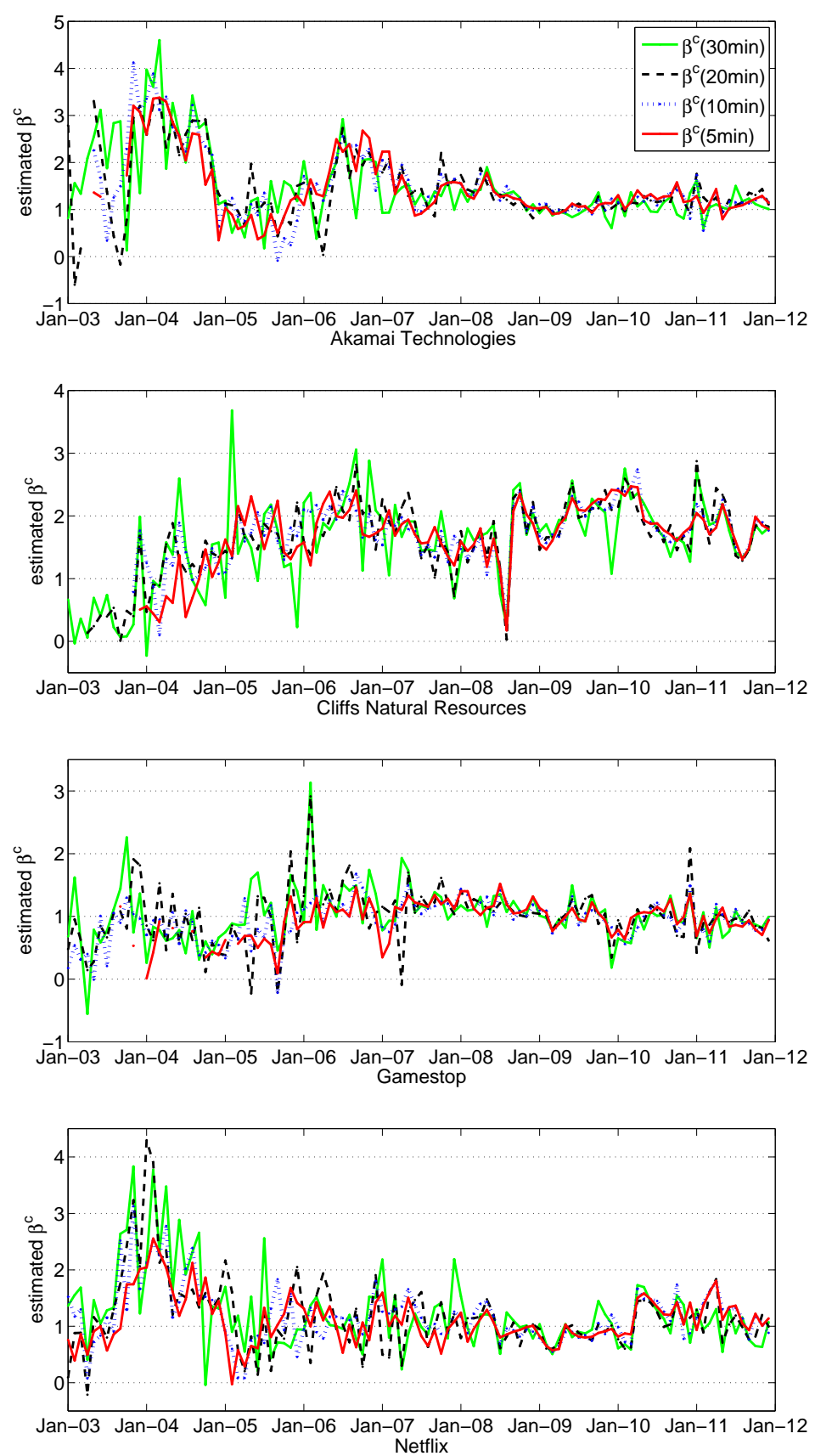
Figure B.9: Beta signature plots for several representative stocks from the S\&P 500 index.

(a) Signature plot for $\beta^{c}$

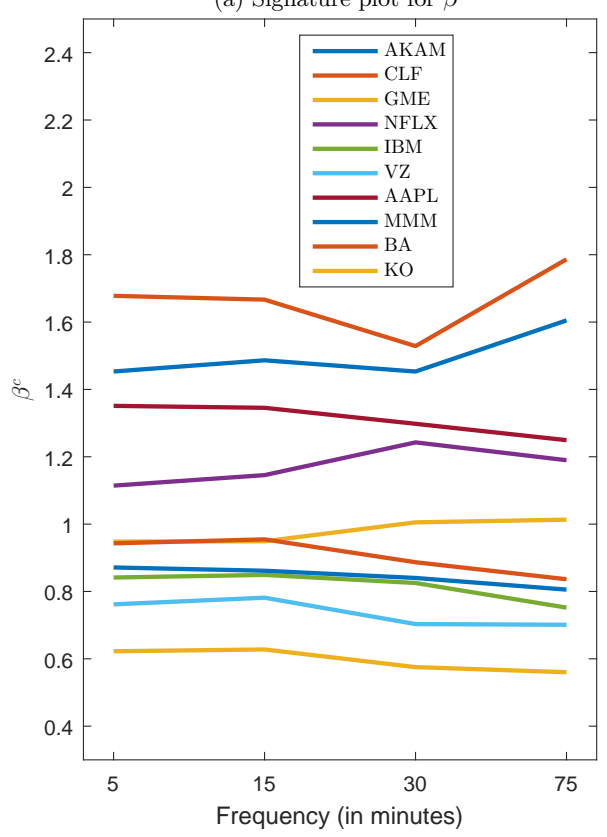

(b) Signature plot for $\beta^{d}$

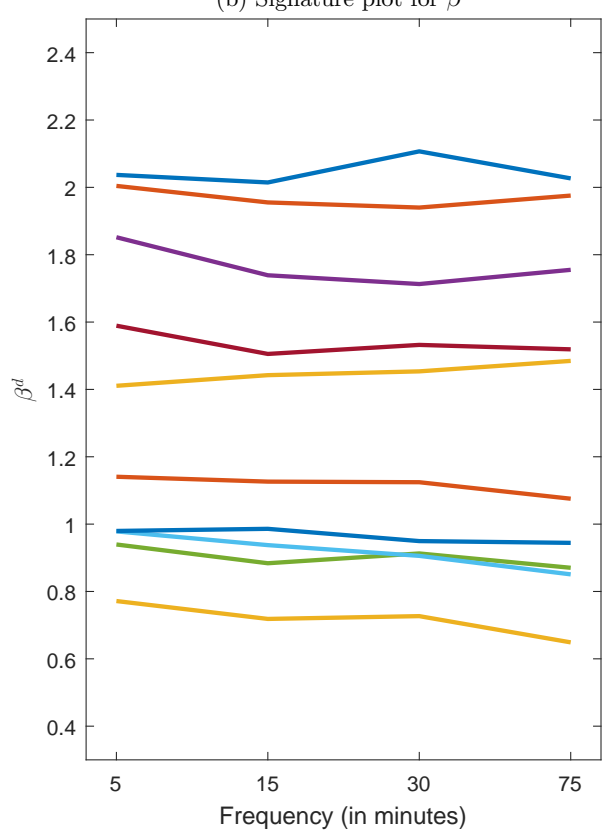

situations where the value of $\alpha_{i}$ is reduced. Results show that the monthly estimates $\hat{\beta}_{i}^{c}$ are very robust to more relaxed truncation thresholds.

The last robustness test is to use different test statistics given by Barndorff-Nielsen and Shephard (2006) for detecting jump days. Apart from the adjusted ratio test $\hat{\mathcal{J}}$, there are two other test statistics which have the same asymptotic distribution:

$$
\begin{aligned}
& \hat{\mathcal{G}}=\frac{1}{\sqrt{\Delta}} \frac{\mu_{1}^{-2} \cdot B V_{0}-R V_{0}}{\phi \mu_{1}^{-4} \cdot D V_{0}} \stackrel{L}{\longrightarrow} \mathcal{N}(0,1), \\
& \hat{\mathcal{H}}=\frac{1}{\sqrt{\Delta}} \frac{1}{\sqrt{\phi \cdot D V_{0} / B V_{0}^{2}}}\left(\frac{\mu_{1}^{-2} \cdot B V_{0}-R V_{0}}{R V_{0}}\right) \stackrel{L}{\longrightarrow} \mathcal{N}(0,1) .
\end{aligned}
$$

The difference statistic $\hat{\mathcal{G}}$ and the ratio statistic $\hat{\mathcal{H}}$ in general detect more jump days than the adjusted ratio statistic $\hat{\mathcal{J}}$ in equation (17). For the equally weighted portfolio that we constructed, using $\hat{\mathcal{G}}$ leads to 314 jump days which are contained in 98 months, and using $\hat{\mathcal{H}}$ leads to the same 161 jump days which are contained in 82 months. Most of the corresponding $\hat{\beta}_{i}^{d \prime}$ s do not alter up to one decimal place.

\section{Acknowledgements}

We are grateful for comments from Torben Andersen, Eser Arisoy, Andrew Patton, George Tauchen, Viktor Todorov and participants at the 2013 Midwest Econometrics Group Meeting, SIRCA 
Young Researcher Workshop, Auckland Finance Meeting, 2014 China Meeting of the Econometric Society, and the Econometric Society Australasian Meeting. This research is supported by funding from ARC DP130100168.

\section{References}

Aït-Sahalia, Y. and Jacod, J. (2012), 'Analyzing the spectrum of asset returns: Jump and volatility components in high frequency data', J. Econ. Lit. 50(4), 1007-50.

Anand, Amber, A., Irvine, R., Puckett, A. and Venkataraman, K. (2013), 'Institutional trading and stock resiliency: Evidence from the 2007-2009 financial crisis', J. Finan. Econ. 108, 773-797.

Andersen, T. G., Bollerslev, T. and Diebold, F. X. (2007), 'Roughing it up: Including jump components in the measurement, modeling, and forecasting of return volatility', Rev. Econ. Stat. 89(4), 701-720.

Andersen, T. G., Bollerslev, T., Diebold, F. X. and Labys, P. (2003), 'Modelling and forecasting realized volatility', Econometrica 71, 579-625.

Bandi, R. and Russell, J. (2006), 'Separating microstructure noise from volatility', J. Finan. Econ. 79, 655-692.

Barndorff-Nielsen, O. E. and Shephard, N. (2006), 'Econometrics of testing for jumps in financial economics using bipower variation', J. Financ. Econ. 4(1), 1-30.

Beber, A. and Pagano, M. (2013), 'Short-selling bans around the world: Evidence from the 2007-09 crisis', J. Financ. 68(1), 343-381.

Black, A., Chen, J., Gustap, O. and Williams, J. M. (2012), The importance of jumps in modelling volatility during the 2008 financial crisis, Technical report.

Blair, B., Poon, S.-H. and Taylor, S. (2001), 'Forecasting s\&p500 volatility: The incremental information content of implied volatilities and high-frequency index returns', J. Econ. 105, 5-26.

Bollerslev, T., Law, T. H. and Tauchen, G. (2008), 'Risk, jumps, and diversification', J. Econ. 144(1), 234-256.

Bollerslev, T., Li, S. Z. and Todorov, V. (2016), 'Roughing up beta: Continuous versus discontinuous betas and the cross section of expected stock returns', Journal of Financial Economics 120(3), 464-490.

Campbell, J. Y., Hilscher, J. and Szilagyi, J. (2008), 'In search of distress risk', J. Financ. 63(6), 28992939.

Chan, K. C. and Chen, N.-F. (1991), 'Structural and return characteristics of small and large firms', J. Financ. 46(4), 1467-1484.

Dungey, M., Luciani, M. and Veredas, D. (2012), Ranking systemically important financial institutions, Working Papers ECARES 2013/130530, ULB - Universite Libre de Bruxelles.

Dungey, M., McKenzie, M. and Smith, V. (2009), 'Empirical evidence on jumps in the term structure of the us treasury market', J. Empir. Financ. 16, 430-445.

Epps, T. W. (1979), 'Comovements in stock prices in the very short run', J. Amer. Stat. Assoc. 74(366), 291-298. 
Fama, E. F. and MacBeth, J. D. (1973), 'Risk, return, and equilibrium: Empirical tests', J. Polit. Econ. 81(3), 607-636.

Ferson, W. E. and Harvey, C. R. (1991), 'The variation of economic risk premiums', J. Polit. Econ. 99(2), 385-415.

Giordani, P. and Kohn, R. (2008), 'Efficient bayesian inference for multiple change-point and mixture innovation models', J. Bus. Econ. Stat. 26(1), 66-77.

Hanson, F. (2007), Applied Stochastic Processes and Control for Jump Diffusions: Modeling, Analysis, and Computation, Advances in Design and Control, Society for Industrial and Applied Mathematics.

Hoberg, G. and Phillips, G. (2010), 'Real and financial industry booms and busts', J. Financ. 65(1), 4586.

Jacod, J. and Todorov, V. (2009), 'Testing for common arrivals of jumps for discretely observed multidimensional processes', Ann. Stat. 37(4), 1792-1838.

Jarrow, R. A. and Rosenfeld, E. R. (1984), 'Jump risks and the intertemporal capital asset pricing model', The Journal of Business 57(3), 337-351.

URL: $h$ ttp://www.jstor.org/stable/2352866

Lahaye, J., Laurent, S. and Neely, C. J. (2005), 'Bond yields and the federal reserve', J. Polit. Econ. 113(2), 311-344.

Lahaye, J., Laurent, S. and Neely, C. J. (2011), 'Jumps, cojumps and macro announcements', J. Appl. Econ. 26(6), 893-921.

Lai, Y.-S. and Sheu, H.-J. (2010), 'The incremental value of a futures hedge using realized volatility', J. Futur. Mark. 30(9), 874-896.

Patton, A. J. and Verardo, M. (2012), 'Does beta move with news? firm-specific information flows and learning about profitability', Rev. Financ. Stud. 25, 2789-2839.

Roll, R. (1977), 'A critique of the asset pricing theory's tests part i: On past and potential testability of the theory', J. Finan. Econ. 4(2), $129-176$.

Roll, R. and Ross, S. A. (1994), 'On the cross-sectional relation between expected returns and betas', J. Financ. 49(1), 101-121.

Sharpe, W. F. (1963), 'A simplified model for portfolio analysis', Management Science 9(2), 277 - 293.

Todorov, V. and Bollerslev, T. (2010), 'Jumps and betas: A new framework for disentangling and estimating systematic risks', J. Econ. 157(2), 220-235. 